\title{
Efficacy and Safety of Disinfectants for Decontamination of N95 and SN95 Filtering Facepiece Respirators: Protocol for a Systematic Review
}

PRISMA-P (Preferred Reporting Items for Systematic review and Meta-Analysis Protocols) 2015 checklist Mar 24,2020

\begin{tabular}{ccc}
\hline Section and topic & $\begin{array}{c}\text { Item } \\
\text { No }\end{array}$ & Checklist item \\
\hline
\end{tabular}

Title:

Identification 1a Identify the report as a protocol of a systematic review

Update $\quad 1 b$ If the protocol is for an update of a previous systematic review, identify as such

\section{ADMINISTRATIVE INFORMATION}

Efficacy and Safety of Disinfectants for the Decontamination of N95 and SN95 Filtering Facepiece Respirators: Protocol for a Systematic Review

This is an original review, not an update

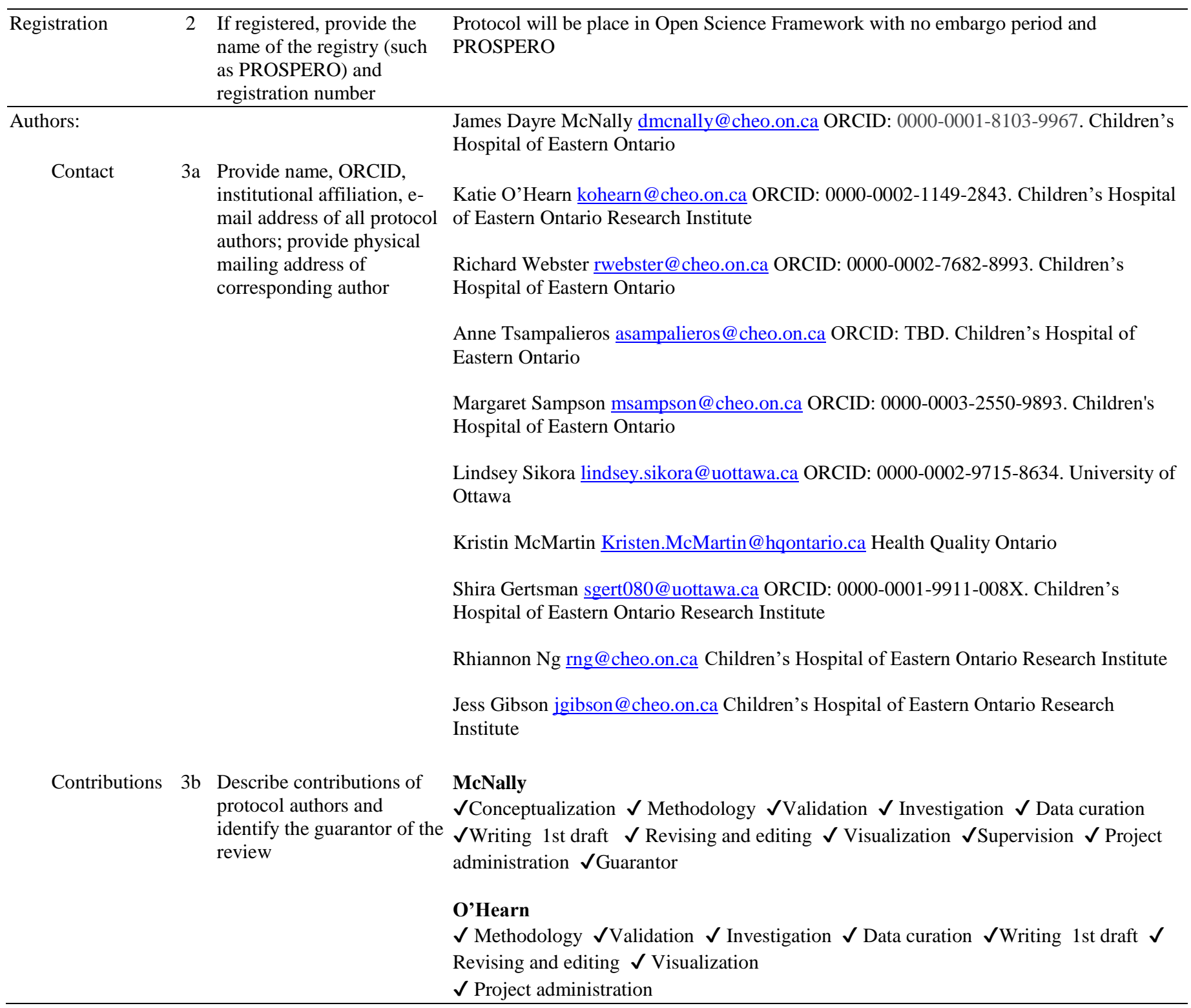




\section{Webster}

$\boldsymbol{\checkmark}$ Methodology $\sqrt{ }$ Validation $\boldsymbol{\checkmark}$ Investigation $\boldsymbol{\checkmark}$ Data curation $\boldsymbol{\checkmark}$ Writing 1st draft $\boldsymbol{\checkmark}$ Revising and editing $\checkmark$ Visualization

Tsampalieros $\checkmark$ Methodology $\checkmark$ Validation $\checkmark$ Investigation $\checkmark$ Data curation $\checkmark$ Writing 1st draft $\checkmark$ Revising and editing $\checkmark$ Visualization

\section{Sampson}

$\checkmark$ Methodology $\boldsymbol{\checkmark}$ Formal analysis $\boldsymbol{\checkmark}$ Investigation $\boldsymbol{\checkmark}$ Data curation $\boldsymbol{\checkmark}$ Revising and editing $\checkmark$ Visualization $\checkmark$ Project administration

\section{Sikora}

$\checkmark$ Methodology $\sqrt{ }$ Formal analysis $\sqrt{ }$ Investigation

$\checkmark$ Data curation $\checkmark$ Revising and editing $\checkmark$ Visualization $\checkmark$ Project administration

\section{McMartin}

$\checkmark$ Revising and editing

\section{Gertsman}

$\checkmark$ Methodology $\boldsymbol{\checkmark}$ Validation $\checkmark$ Investigation $\checkmark$ Data curation $\boldsymbol{\checkmark}$ Writing 1 st draft $\checkmark$ Revising and editing $\sqrt{ }$ Visualization

$\mathrm{Ng}$

$\checkmark$ Data curation $\checkmark$ Revising and editing

Gibson

$\checkmark$ Data curation $\checkmark$ Revising and editing

\begin{tabular}{|c|c|c|c|}
\hline Amendments & 4 & $\begin{array}{l}\text { If the protocol represents an } \\
\text { amendment of a previously } \\
\text { completed or published } \\
\text { protocol, identify as such } \\
\text { and list changes; otherwise, } \\
\text { state plan for documenting } \\
\text { important protocol } \\
\text { amendments }\end{array}$ & $\begin{array}{l}\text { The COVID-19 literature is expanding rapidly with over } 2000 \text { publications by mid-March } \\
\text { 2020. Protocol amendments are likely and will be documented in OSF. }\end{array}$ \\
\hline \multicolumn{4}{|l|}{ Support: } \\
\hline Sources & $5 \mathrm{a}$ & $\begin{array}{l}\text { Indicate sources of financial } \\
\text { or other support for the } \\
\text { review }\end{array}$ & This research has received no specific funding \\
\hline Sponsor & $5 b$ & $\begin{array}{l}\text { Provide name for the review } \\
\text { funder and/or sponsor }\end{array}$ & This research does not have a funder or sponsor \\
\hline \multirow[t]{2}{*}{$\begin{array}{l}\text { Role of } \\
\text { sponsor or } \\
\text { funder }\end{array}$} & $5 \mathrm{c}$ & $\begin{array}{l}\text { Describe roles of funder(s), } \\
\text { sponsor(s), and/or } \\
\text { institution(s), if any, in } \\
\text { developing the protocol }\end{array}$ & This research does not have a funder or sponsor \\
\hline & & & INTRODUCTION \\
\hline Rationale & 6 & $\begin{array}{l}\text { Describe the rationale for } \\
\text { the review in the context of } \\
\text { what is already known }\end{array}$ & $\begin{array}{l}\text { During the COVID-19 pandemic, a shortage of personal protective equipment (PPE), } \\
\text { namely surgical masks, N95 masks, and gowns, has been experienced by some hospitals } \\
\text { and could be expected in others due to a rapidly increased need. One method of } \\
\text { addressing the shortage is to decontaminate and re-use PPE. The Centres for Disease } \\
\text { Control (CDC) specifically recommends N95 filtering facepiece respirators (FFRs) for } \\
\text { healthcare workers who are interacting with patients with COVID-19. There are anecdotal } \\
\text { reports and published literature evaluating the potential of using disinfectants, such as } \\
\text { hydrogen peroxide and bleach to decontaminate FFRs, with mixed reports of impact on } \\
\text { structural integrity. To date this literature has not been comprehensively synthesized and }\end{array}$ \\
\hline
\end{tabular}


the purpose of this review is to systematically review the existing literature on the use of disinfectants for the decontamination of facemask PPE.

This information will be used to contribute to FFR decontamination protocols at the Children's Hospital of Eastern Ontario and shared with other hospitals in Ontario, Canada, and internationally.

\begin{tabular}{lll}
\hline Objectives & $7 \begin{array}{l}\text { Provide an explicit } \\
\text { statement of the question(s) } \\
\text { the review }\end{array}$ & $\begin{array}{l}\text { The objective of this review is to identify and synthesize data from published studies } \\
\text { and SN95 FFR. }\end{array}$ \\
& will address with reference \\
& to participants, \\
& interventions, comparators, \\
& and outcomes (PICO)
\end{tabular}

METHODS

Eligibility criteria $\quad 8 \quad$ Specify the study

Population: N95 filtering face masks (particulate and surgical) or their components characteristics (such as Exposure: Disinfecants, including hydrogen peroxide (vapour, liquid, gas plasma), bleach PICO, study design, setting, (sodium hypochlorite, submersion, wipe), ethanol, isopropyl alcohol, ethylene oxide time frame) and report characteristics (such as Comparison: There is no set comparison group. Stand-alone criteria have been set for characteristics (such as years considered, langu
publication status) to be used as criteria for mask performance (penetration, flow resistance) and we will primarily compare against eligibility for the review

Outcome: Studies that report on at least one of the following outcomes will be included. Outcomes will be reported separately for each type of disinfectant.

1. Impact of each disinfectant decontamination method on FFR performance, with a specific focus on aerosol penetration and airflow resistance

2. Effectiveness of each disinfectant at removing viral or bacterial load

3. Measures or observations related to change in fit or physical degradation following exposure to each disinfectant

4. User safety and comfort, including odour and irritation following exposure to each disinfectant.

Study designs: Original publications examining the use of disinfectants including hydrogen peroxide (vapour, liquid, gas plasma), bleach (sodium hypochlorite), ethanol, isopropyl alcohol, and ethylene oxide to decontaminate N95 FFRs in English or French published after 1972 will be included in this review. We will also include systematic reviews of disinfectant treatment of N95 FFRs. Editorial reviews, narrative reviews, patents and books will be excluded.

\begin{tabular}{|c|c|c|c|}
\hline $\begin{array}{l}\text { Information } \\
\text { sources }\end{array}$ & 9 & $\begin{array}{l}\text { Describe all intended } \\
\text { information sources (such as } \\
\text { electronic databases, contact } \\
\text { with study authors, trial } \\
\text { registers or other grey } \\
\text { literature sources) with } \\
\text { planned dates of coverage }\end{array}$ & $\begin{array}{l}\text { Ovid Medline, Ovid Embase, Global Health, Google Scholar, WHO COVID-19 feed, } \\
\text { MedRxiv, Scopus for targeted authors were searched. } \\
\text { Two journals will be handsearched (as they are not indexed in any databases): Journal of } \\
\text { the International Society for Respiratory Protection, and the Journal of Engineered Fibers } \\
\text { and Fabrics. } \\
\text { Reference Lists of eligible studies will be examined. } \\
\text { Searches will cover the period 1972, year of introduction of the N95- present. } \\
\text { Disaster Lit: Database for Disaster Medicine and Public on the National Library of } \\
\text { Medicine will also be searched. }\end{array}$ \\
\hline
\end{tabular}




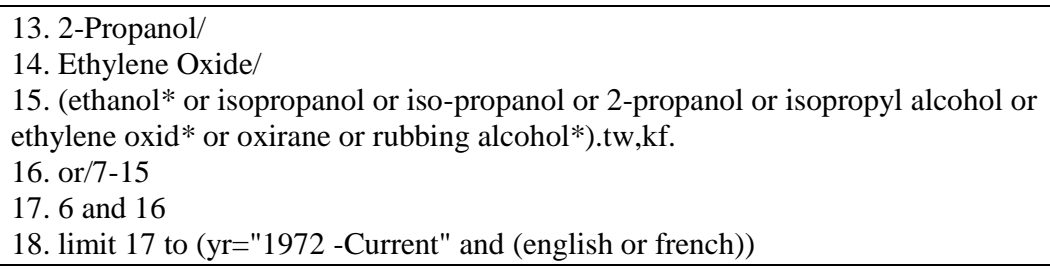

Study records:
Data
management
Selection
process


Data
collection
process

Data items

11a Describe the mechanism(s) Records will be downloaded into Endnote for duplicate removal and exported to that will be used to manage InsightScope (www.insightscope.ca) for screening. Data abstraction will be performed records and data throughout using REDCap. the review

$11 \mathrm{~b}$ State the process that will be Studies will be selected by 2 levels of screening (title and abstract, full text) against used for selecting studies inclusion criteria by at least 2 independent reviewers reaching consensus. and/or (such as two independent resolution by the lead author. reviewers) through each phase of the review (that is, screening, eligibility and inclusion in meta-analysis)

11c Describe planned method of A test set of at least 30 citations will be used to evaluate the inclusion/exclusion criteria. extracting data from reports The test set will be created by a member of the investigative team and verified by a (such as piloting forms, second. All reviewers who contribute to screening will first perform the test set, and must done independently, in achieve a sensitivity of $\geq 80 \%$ before they are given access to the study records. Reviewers duplicate), any processes for who do not achieve $\geq 80 \%$ sensitivity on the test set will be provided with additional obtaining and confirming training and repeat a second test set. If $\geq 80 \%$ sensitivity is achieved on the repeat test set, data from investigators the reviewer will be given access to the study records.

The data abstraction forms in REDCap will be piloted by at least two members of the investigative team against a total of 6 eligible studies.

12 List and define all variables Study demographics:

for which data will be sought (such as PICO items, funding sources), any preplanned data assumptions and simplifications

- Authors and contact information

- Title

- Country

- Journal and year of publication

Study Design:

- $\quad$ Single vs. multiple decontamination protocols

- $\quad$ Pre and post, post only

- Controlled vs uncontrolled

- Number of experimental conditions

PPE tested

- $\quad$ Mask type and model

- $\quad$ Full mask or components

Decontamination Method

- $\quad$ Agent used, solution concentration, original concentration (manufacturing specification), duration of exposure, type of exposure (i.e. submersion, gassing), number of cycles

- Method of application

- Method of supporting/suspending masks

- Drying/post-decontamination processing

- Implementation of the decontamination process

Aerosol Penetration Test used (including sizes evaluated)

Airflow Resistance Test

Methods of pathogen contamination and extraction

Outcomes

- $\quad$ Penetration (absolute percentage, change relative to baseline)

- $\quad$ Airflow resistance and pressure drop

- Absolute and relative change in pathogen number

- $\quad$ Fit Testing: ability to achieve appropriate fit, particle loss

- Observations of physical degradation 


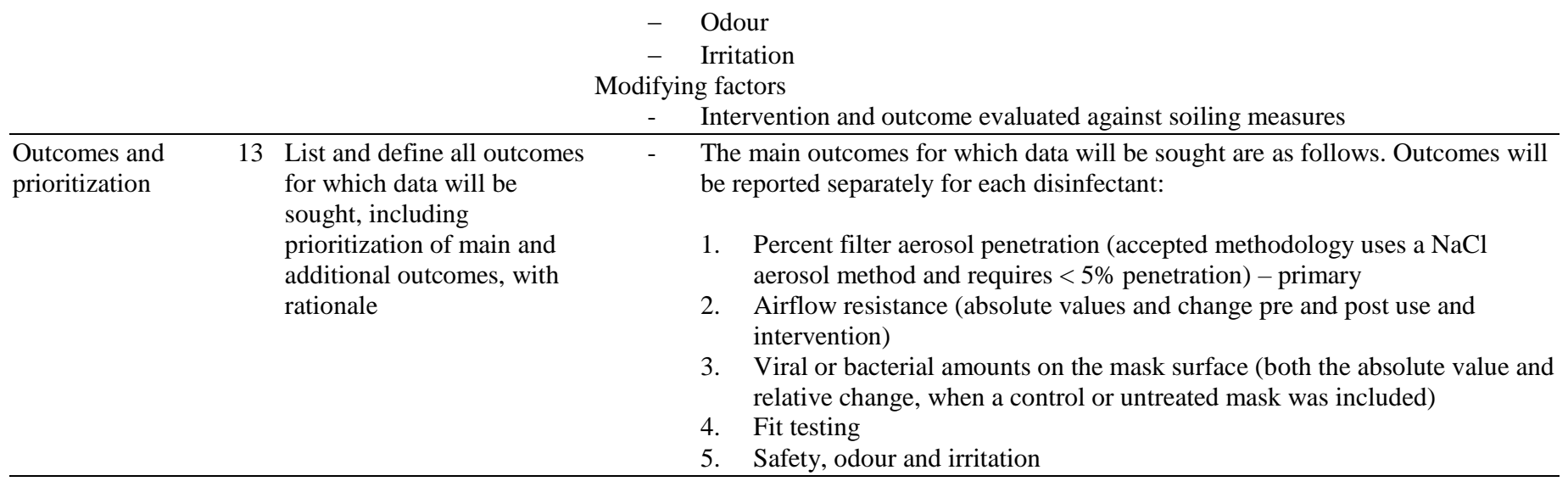

\begin{tabular}{|c|c|c|c|}
\hline & 14 & $\begin{array}{l}\text { Describe anticipated } \\
\text { methods for assessing risk } \\
\text { of bias of individual studies, } \\
\text { including whether this will } \\
\text { be done at the outcome or } \\
\text { study level, or both; state } \\
\text { how this information will be } \\
\text { used in data synthesis }\end{array}$ & $\begin{array}{l}\text { Risk of bias for each outcome will be ascertained at the study level and reported } \\
\text { in the manuscript. The impact of risk of bias on the summarized evidence will be } \\
\text { addressed in the discussion section of the manuscript. } \\
\text { Risk of bias will be ranked as low, moderate, or high for each of the following } \\
\text { criteria (examples of low risk in parentheses): } \\
\text { - Design (controlled or pre-post) } \\
\text { - Methodological consistency (same procedures/conditions across study } \\
\text { - Population heterogeneity (masks obtained from same lots and models } \\
\text { - } \text { evaluated separately) } \\
\text { - Sampling bias (all missing data accounted for) } \\
\text { - Selective reporting (results for all objective reported) }\end{array}$ \\
\hline
\end{tabular}

Data synthesis 15a Describe criteria under which study data will be 1 . quantitatively synthesised

$15 b$ If data are appropriate for quantitative synthesis, describe planned summary measures, methods of handling data and methods of combining data from studies, including any planned exploration of consistency (such as $\mathrm{I}^{2}$, Kendall's $\tau$ )

15c Describe any proposed additional analyses (such as
1. Penetration and airflow outcomes: For each given disinfectant, data will need to be available from at least three independent studies using approved testing methods to consider quantitative synthesis.

2. Bacterial decontamination: Significant heterogeneity is expected in methodology related to pathogen type (virus vs bacteria), medium, application method, and method of evaluation of decontamination. For each disinfectant, we will quantitatively synthesize this outcome if similar data is provided from 3 studies using concordant pathogen and testing.

For both the particle penetration and airflow resistance outcomes the data is anticipated to be primarily presented as an absolute value. If presented as a percent change, and sufficient data is provided, we will calculate the absolute value. Prior to pooling we will perform tests of statistical heterogeneity using $\mathrm{I}^{2}$ tests. Random effects meta-analysis will be employed to present the pooled absolute value disinfectant treatment and relative change (from control or no treatment arm).

Significant heterogeneity could be anticipated with the methods and outcomes related to contamination and decontamination. To account for differences in methods (viral load, application and retrieval from mask) we will report and pool results using log change (in addition to absolute value or change). Again, prior to pooling the results we will perform tests of statistical heterogeneity using $\mathrm{I}^{2}$ tests. Random effects meta-analysis will be utilized.

Subgroup/sensitivity analyses: 
sensitivity or subgroup analyses, meta-regression)

$15 \mathrm{~d}$ If quantitative synthesis is not appropriate, describe the type of summary planned

Meta-bias(es)

16 Specify any planned assessment of meta-bias(es) (such as publication bias across studies, selective reporting within studies)

Confidence in cumulative evidence
17 Describe how the strength of the body of evidence will be assessed (such as GRADE)
1. The primary indication for this work is the current pandemic related to SarsCoV-2. For the synthesis and presentation of decontamination results we perform a separate analysis limiting to studies that use viral pathogens. This data will be analysed and reported separately for each disinfectant.

2. If sufficient data permits we will describe and quantitatively synthesize the data for low risk studies (again $>=3$ studies)

3. Differential impact of each disinfectant treatment by FFR mask type could be anticipated. If FFR performance is observed to be reduced below acceptable for any mask, we will present and synthesize the results by specific mask type heterogeneous, the study findings will be presented descriptively in tables and text. Based on the anticipated small size of the available literature (approximately 10 studies) ) and limited variability in study sample sizes generally observed in laboratory studies, no statistical test of publication bias are planned.

The cumulative body of evidence for each pre-defined outcome (impacts of each disinfectant on particle penetration, airflow resistance, germicidal effect, fit, physical changes, potential health risks) will be evaluated using risk of bias and consistency.

Conclusions will be considered consistent for each individual outcome if equivalent outcome valences (i.e. success or failure according to pre-determined standards) are reported in at least two studies with no contrasting results in any other study.

Template source: Shamseer L, Moher D, Clarke M, Ghersi D, Liberati A, Petticrew M, Shekelle P, Stewart L, PRISMA-P Group.

Preferred reporting items for systematic review and meta-analysis protocols (PRISMA-P) 2015: elaboration and explanation.

BMJ. 2015 Jan 2;349(jan02 1):g7647. 